\title{
Efficacy of tumor-targeting Salmonella typhimurium A1-R in combination with anti-angiogenesis therapy on a pancreatic cancer patient-derived orthotopic xenograft (PDOX) and cell- line mouse models
}

\author{
Yukihiko Hiroshima ${ }^{1,2,3}$, Yong Zhang ${ }^{1}$, Takashi Murakami ${ }^{3}$, Ali Maawy ${ }^{2}$, Shinji \\ Miwa $^{1,2}$, Mako Yamamoto ${ }^{1,2}$, Shuya Yano ${ }^{1,2}$, Sho Sato ${ }^{3}$, Masashi Momiyama ${ }^{3}$, \\ Ryutaro Mori, Ryusei Matsuyama ${ }^{3}$, Takashi Chishima ${ }^{3}$, Kuniya Tanaka ${ }^{3}$, Yasushi \\ Ichikawa $^{3}$, Michael Bouvet ${ }^{2}$, Itaru Endo ${ }^{3}$, Ming Zhao ${ }^{1}$ and Robert M. Hoffman ${ }^{1,2}$ \\ ${ }^{1}$ AntiCancer, Inc., San Diego, CA, USA \\ 2 Department of Surgery, University of California San Diego, San Diego, CA, USA \\ ${ }^{3}$ Yokohama City University Graduate School of Medicine, Yokohama, Japan \\ Correspondence to: Robert M. Hoffman or Ming Zhao, email: all@anticancer.com \\ Keywords: Pancreatic cancer, Salmonella typhimurium A1-R, patient-derived orthotopic xenograft (PDOX), orthotopic, nude \\ mice, GFP, VEGF, anti-angiogenic therapy, bevacizumab, gemcitabine \\ Received: September 17,2014 Accepted: October 28, $2014 \quad$ Published: October 28, 2014
}

This is an open-access article distributed under the terms of the Creative Commons Attribution License, which permits unrestricted use, distribution, and reproduction in any medium, provided the original author and source are credited.

\section{ABSTRACT}

The aim of the present study was to examine the efficacy of tumor-targeting Salmonella typhimurium A1-R treatment following anti-vascular endothelial growth factor (VEGF) therapy on VEGF-positive human pancreatic cancer. A pancreatic cancer patient-derived orthotopic xenograft (PDOX) that was VEGF-positive and an orthotopic VEGF-positive human pancreatic cancer cell line (MiaPaCa-2-GFP) as well as a VEGFnegative cell line (Panc-1) were tested. Nude mice with these tumors were treated with gemcitabine (GEM), bevacizumab (BEV), and S. typhimurium A1-R. BEV/GEM followed by $S$. typhimurium A1-R significantly reduced tumor weight compared to BEV/GEM treatment alone in the PDOX and MiaPaCa-2 models. Neither treatment was as effective in the VEGF-negative model as in the VEGF-positive models. These results demonstrate that $S$. typhimurium A1-R following anti-angiogenic therapy is effective on pancreatic cancer including the PDOX model, suggesting its clinical potential.

\section{INTRODUCTION}

Pancreatic cancer is one of the most aggressive malignant tumors with a $23 \% 1$-year survival rate and $<2 \%$ 5-year survival rate. The two most commonly used chemotherapy drugs approved for the treatment of pancreatic cancer are gemcitabine (GEM) and 5-fluorouracil (5-FU). In recent years, little progress has been made in understanding and treatment of this disease [1].

In pancreatic cancer, overexpression of vascular endothelial growth factor (VEGF) and its receptors is associated with poor prognosis and increased metastatic potential [2, 3]. Bevacizumab (BEV) is a humanized monoclonal VEGF-neutralizing antibody that many tumors become resistant to after a short period of response
[4].

Our laboratory has previously developed a genetically modified bacterial strain, Salmonella typhimurium A1, selected for anticancer activity in vivo. S. typhimurium A1 is auxotrophic (leu/arg-dependent) [5]. The strain targets and grows in tumors. In contrast, normal tissue is cleared of these bacterial even in immunodeficient athymic mice. In order to increase the tumor-targeting capability of A1, the strain was re-isolated after infection of a human colon tumor growing in nude mice. The tumor-isolated strain, termed $S$. typhimurium A1-R, had increased targeting for cells in vivo as well as in vitro [6].

S. typhimurium A1-R is effective against prostate cancer [7], breast cancer [6, 8], pancreatic cancer [9-12], glioma [13, 14], lung cancer [15], fibrosarcoma [16] and osteosarcoma [17]. 
In the present study, we demonstrate the efficacy of $S$. typhimurium A1-R following antiangiogenic therapy with bevacizumab/gemcitabine (BEV/GEM) in patientderived orthotopic xenograft (PDOX) and cell line nudemouse models of pancreatic cancer.

\section{RESULTS AND DISCUSSION}

\section{Differential expression patterns of VEGF-related genes in pancreatic cancer cell lines}

In order to identify potential BEV-sensitive pancreatic cancer cell lines, mRNA expression of $V E G F A$, VEGFR1 and VEGFR2 in pancreatic cancer cell lines (BxPC-3, Capan-1, Hs766T, MiaPaCa-2 and Panc-1) was examined by real-time RT-PCR (Fig. 1). MiaPaCa-2 expressed VEGFA significantly more than other cell lines $(\mathrm{p}<0.001)$ except for BxPC-3 ( $=0.558)$ (Fig. 1A). MiaPaCa-2 expressed VEGFR2 significantly more than other cell lines (BxPC-3: $\mathrm{p}=0.005$; Capan-1: $\mathrm{p}<0.001$; Hs766T: $\mathrm{p}=0.005$; and Panc-1: $\mathrm{p}=0.006$ ) (Fig. 1C). VEGFR1 expression was not detected in MiaPaCa-2 and Capan-1 cell lines (Fig. 1B).

\section{S. typhimurium A1-R killed MiaPaCa-2 and Panc- 1 pancreatic cancer cells in vitro}

GFP-expressing $S$. typhimurium A1-R invaded MiaPaCa-2 and Panc-1 pancreatic cancer cells as early as $60 \mathrm{~min}$, and replicated in the cells $120 \mathrm{~min}$ after infection. Both cancer cell types appeared to die via apoptosis $24 \mathrm{hr}$ after bacterial infection (Fig. 2A). In the clonogenic assay, the average colony area of MiaPaCa- 2 treated with $S$. typhimurium A1-R was $2.95 \pm 0.84 \mathrm{~mm}^{2}$ compared to the untreated control, $6.03 \pm 0.86 \mathrm{~mm}^{2}$. The average colony area of Panc-1 treated with S. typhimurium A1-R was 0.93 $\pm 0.31 \mathrm{~mm}^{2}$, compared to the untreated control, $1.91 \pm 0.10$ $\mathrm{mm}^{2}$. S. typhimurium A1-R significantly reduced colony formation of both pancreatic cancer cell lines compared to the control (MiaPaCa-2: $\mathrm{p}=0.001$ and Panc-1: $\mathrm{p}<0.001$ ) (Fig. 2B and 2C).

\section{Differential sensitivity to $\mathrm{BEV}$ in pancreatic cancer cell lines growing subcutaneously in nude mice}

Real-time RT-PCR of VEGF-related gene expression (Fig. 1) predicted that $\mathrm{MiaPaCa}-2$ was $\mathrm{BEV}$-sensitive and Panc-1 was BEV-resistant. The efficacy of BEV on these cell lines was first determined using a subcutaneous tumor mouse model (Fig. 3). The average tumor volume
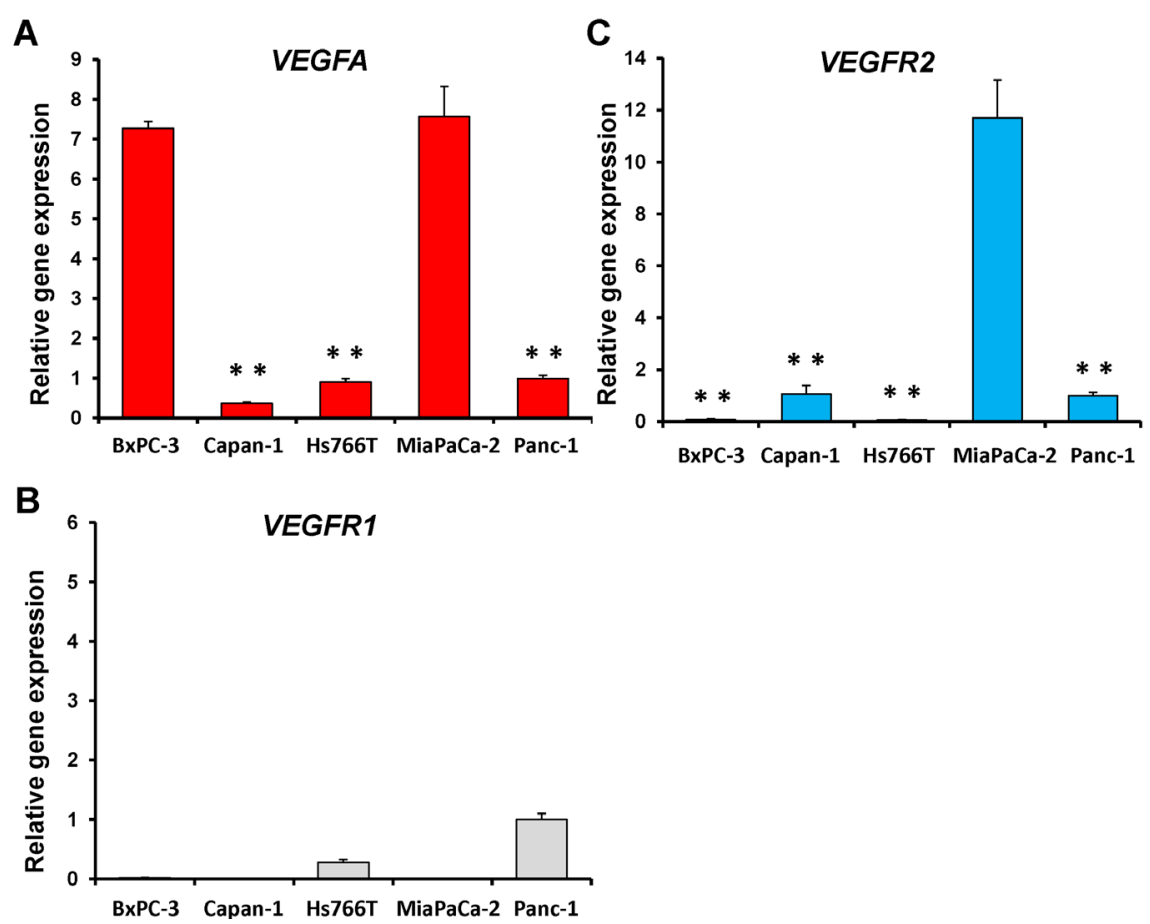

Figure 1: mRNA expression of $\operatorname{VEGFA}(\mathrm{A}), \operatorname{VEGFR1}(\mathrm{B})$ and $V E G F R 2$ (C) in pancreatic cancer cell lines. mRNA expression was determined with real-time RT-PCR. MiaPaCa-2 significantly expressed VEGFA more than the other cell lines $(\mathrm{p}<0.001)$ except for BxPC-3 ( $\mathrm{p}=0.558)(\mathrm{A})$. MiaPaCa-2 significantly expressed VEGFR2 more than other cell lines $(\mathrm{BxPC}-3$ : $\mathrm{p}=0.005$, Capan-1: $\mathrm{p}<0.001$; Hs766T: $\mathrm{p}=0.005$; and Panc-1: $\mathrm{p}=0.006)(\mathrm{C})$. VEGFR1 expression was not detected in MiaPaCa-2 and Capan-1 cell lines (B). Data for each treatment are represented as the mean \pm SD. $* * p<0.01$. 
of the MiaPaCa-2 tumors treated with $\mathrm{BEV}$ was $1.04 \pm$ $0.24 \mathrm{~mm}^{3}$ compared to the control which was $4.19 \pm 1.21$ $\mathrm{mm}^{3}$ on Day 22. The average tumor volume of the Panc-1 tumors treated with $\mathrm{BEV}$ was $5.50 \pm 2.62 \mathrm{~mm}^{3}$ compared to the control which was $5.28 \pm 0.99 \mathrm{~mm}^{3}$ on Day 22. BEV significantly reduced the growth of $\mathrm{MiaPaCa}-2$ compared to the untreated control group on Day $22(\mathrm{p}<0.001)$ but did not reduce the growth of Panc-1 (Fig. 3).
Efficacy of BEV on microvessel density in pancreatic cell lines growing subcutaneously in mice

Subcutaneous tumors (MiaPaCa-2 or Panc-1) were treated with BEV (5 mg/kg, twice a week for 2 weeks) and tumor samples were removed 7 days after the last treatment. Frozen sections from each tumor were stained with anti-mouse CD31 antibody, and the MVD was
A
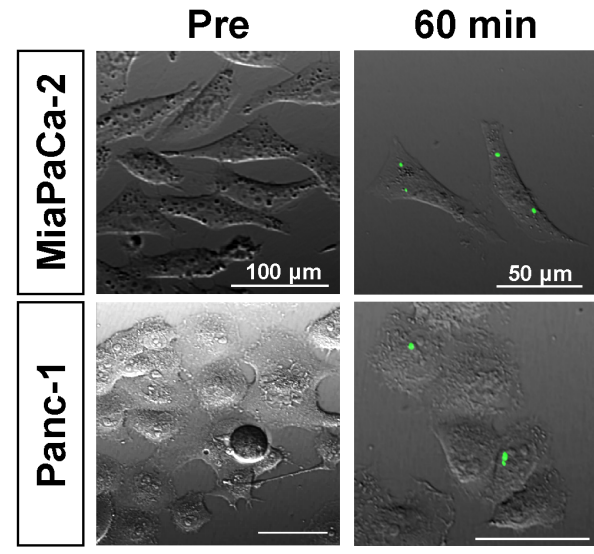

B

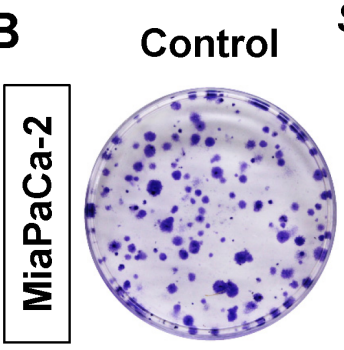

S. typhimurium

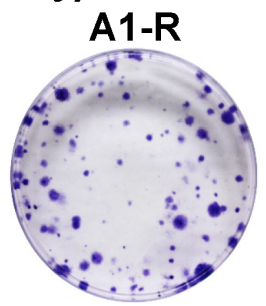

C

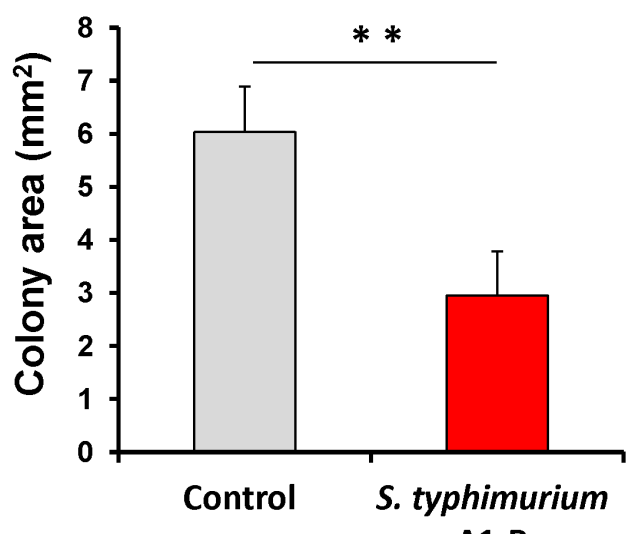

A1-R
$120 \mathrm{~min}$
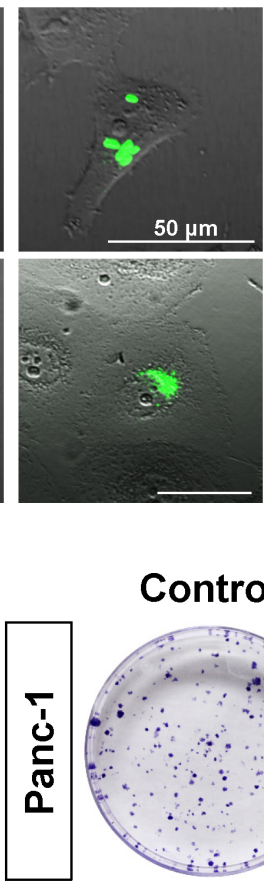

Control

S. typhimurium

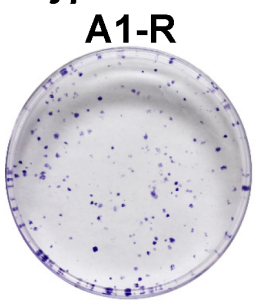

Panc-1

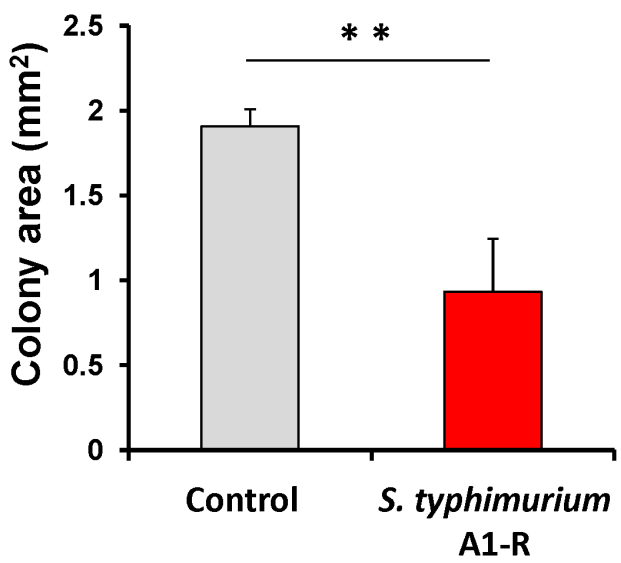

Figure 2: Efficacy of $\boldsymbol{S}$. typhimurium A1-R on pancreatic cancer cell lines. (A) Confocal imaging of MiaPaCa-2 and Panc-1 pancreatic cancer cells infected with S. typhimurium A1-R in vitro. S. typhimurium A1-R infection was detected in both pancreatic cancer cell types after 60 min. S. typhimurium A1-R replicated in the cells after 120 min. S. typhimurium A1-R showed the ability to infect and induce apoptosis in both cell types after $24 \mathrm{hr}$. Scale bars: $100 \mu \mathrm{m}$ (pre); $50 \mu \mathrm{m}$ (60 and $120 \mathrm{~min}) ; 25 \mu \mathrm{m}$ (24 hr). (B and C) MiaPaCa-2 and Panc-1 were treated with $S$. typhimurium A1-R. Clonogenic assays show that $S$. typhimurium A1-R significantly reduced colony formation of both pancreatic cancer cell lines compared to the control groups in vitro (MiaPaCa-2: $\mathrm{p}=0.001$ and Panc-1: $\mathrm{p}<0.001) . * * \mathrm{p}<0.01$. 
determined by counting three fields at $\times 100$ magnification of the highest vascular density. The average MVD of the MiaPaCa-2 tumors treated with $\mathrm{BEV}$ was $27.6 \pm 7.45$ compared to the control which was $65.1 \pm 16.5$. The average MVD of the Panc-1 tumors treated with BEV was $52.4 \pm 8.43$ compared to the control which was $57.4 \pm 5.81$. $\mathrm{BEV}$ significantly reduced the MVD of the MiaPaCa-2 tumor compared to the control $(p=0.002)$ (Fig. 4A, B,
E) but did not significantly reduce MVD of the Panc-1 tumor (Fig. 4C, D, F). These results are consistent with the expression levels of VEGF-related genes (Fig. 1), indicating that MiaPaCa-2 is BEV-sensitive and Panc-1 is BEV-resistant.

A

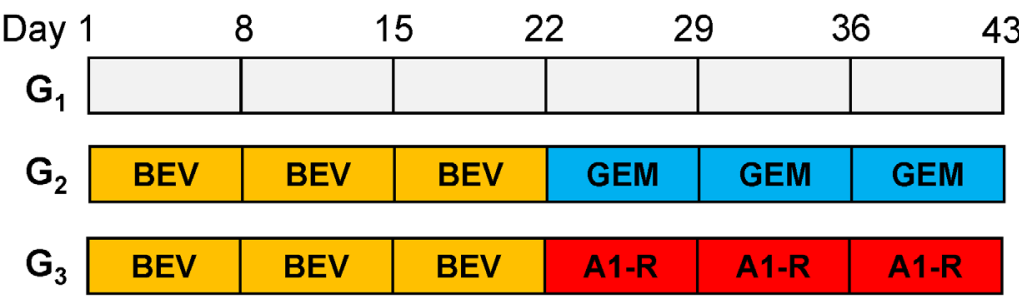

B

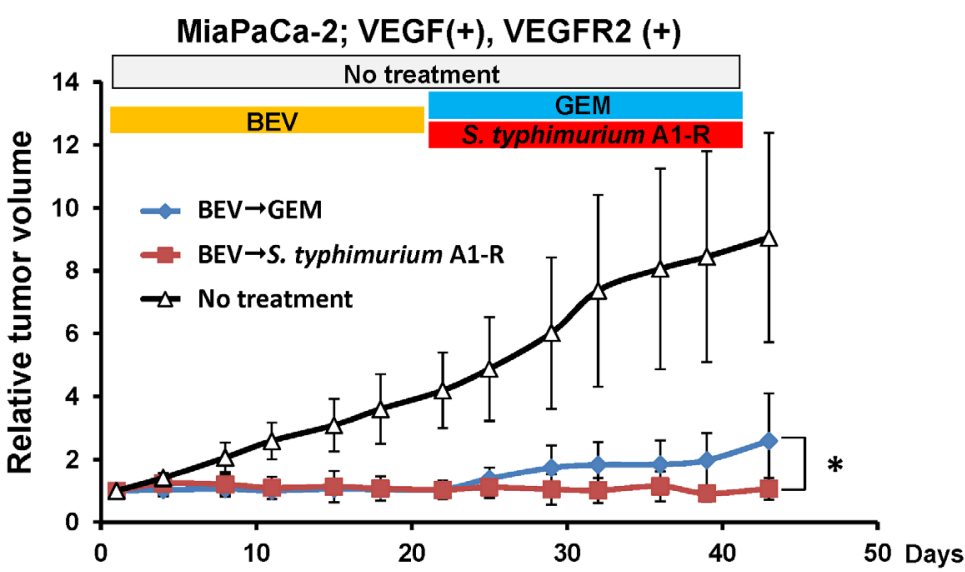

C

Panc-1; VEGF(-), VEGFR2 (-)

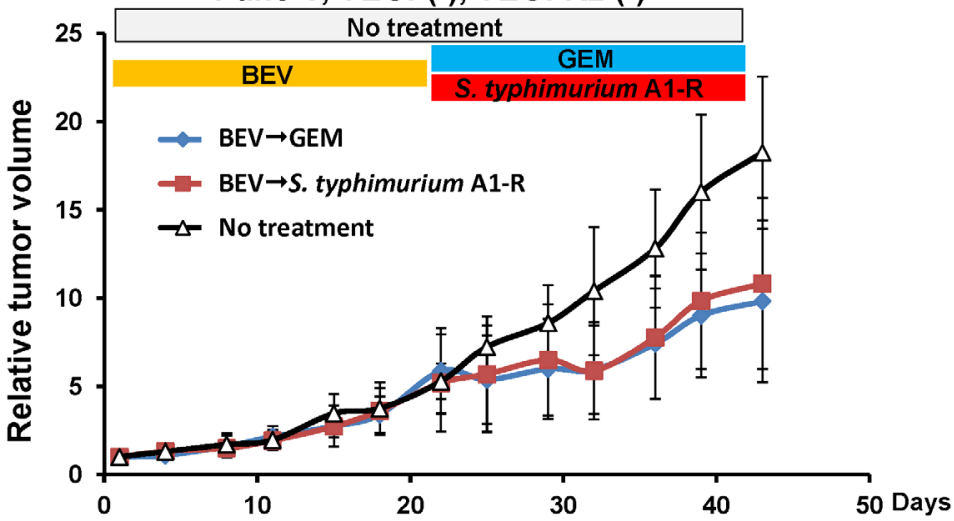

Figure 3: Efficacy of BEV treatment on pancreatic cancer cell lines which have different levels of $V E G F A$ and $V E G F R 2$ expression. (A) Schema of treatments on subcutaneous tumors. Based on RT-PCR results, MiaPaCa-2 was determined to be VEGFApositive and VEGFR2-positive. Panc-1 was determined as VEGFA-negative and VEGFR2-negative. In order to determine the efficacy of $\mathrm{BEV}$ on pancreatic cancer cell lines with different levels of VEGFA and VEGFR2 expression, subcutaneous tumors from MiaPaCa-2 and Panc-1 cells were grown in nude mice and randomized to 3 groups as described in the Materials and Methods. (B) BEV significantly reduced the growth of the MiaPaCa-2 tumor compared to the control on Day $22(\mathrm{p}<0.001)$. Both BEV $\rightarrow$ GEM and BEV $\rightarrow$ S. typhimurium A1-R treatments significantly reduced MiaPaCa-2 tumor growth compared to the control group on Day $43(\mathrm{p}=0.001)$. BEV $\rightarrow$ S. typhimurium A1-R significantly reduced the MiaPaCa-2 tumor growth compared to BEV $\rightarrow$ GEM $(\mathrm{p}=0.037)$. (C) BEV did not reduce the tumor growth of Panc-1 compared to the control on Day 22, but both BEV $\rightarrow$ GEM and BEV $\rightarrow$ S. typhimurium A1-R treatment significantly reduced tumor growth compared to the control on Day $43(\mathrm{BEV} \rightarrow \mathrm{GEM}: \mathrm{p}=0.023$; BEV $\rightarrow$ S. typhimurium A1-R: $\mathrm{p}=0.026) .{ }^{*} \mathrm{p}<0.05$. 


\section{Efficacy of $S$. typhimurium A1-R following BEV treatment on growth of BEV-sensitive and -resistant tumors}

For the BEV-sensitive MiaPaCa-2 tumor, both BEV $\rightarrow$ GEM and BEV $\rightarrow$ S. typhimurium A1-R significantly reduced tumor growth compared to the controls on Day $43(\mathrm{p}=0.001) . \mathrm{BEV} \rightarrow$ S. typhimurium A1-R significantly reduced tumor growth compared to BEV $\rightarrow$ GEM ( $p$
$=0.037)($ Fig. 3B). S. typhimurium A1-R completely suppressed growth of $\mathrm{MiaPaCa}-2$ after $\mathrm{BEV}$ treatment (Fig. 3B). Both BEV $\rightarrow$ GEM and BEV $\rightarrow$ S. typhimurium A1-R significantly reduced tumor growth of the BEVresistant tumor Panc-1 compared to the control (BEV $\rightarrow$ GEM: $\mathrm{p}=0.023 ; \mathrm{BEV} \rightarrow$ S. typhimurium A1-R: $\mathrm{p}=0.026)$ but to a lesser extent than MiaPaCa-2 (Fig. 3C). There was no significant difference in tumor growth inhibition between $\mathrm{BEV} \rightarrow$ S. typhimurium A1-R and BEV $\rightarrow$ GEM.

Furthermore, GFP-labeled S. typhimurium A1-R
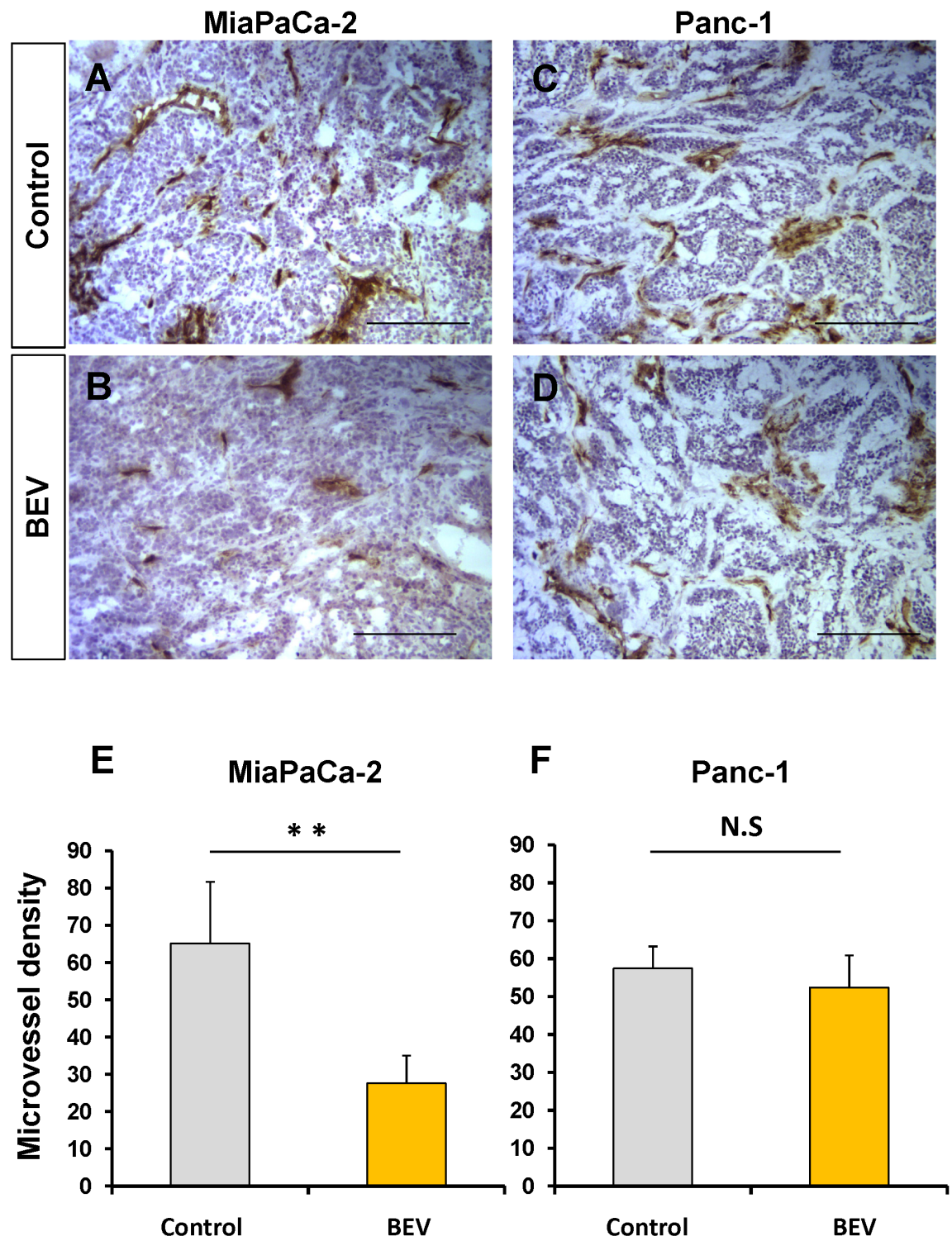

Figure 4: Microvessel density (MVD) in s.c. pancreatic cancer xenografts in nude mice treated with bevacizumab (BEV). To determine MVD, sections were stained with an antibody to CD31 as described in the Materials and Methods. MVD was determined by counting the number of CD31-positive vessels in three fields at $\times 100$ magnification of the highest vascular density. The values are the average numbers of microvessels \pm S.D. (bars) of five different tumors. (A-D) Representative images of frozen sections stained with antiCD31 antibody. The number of vessels in MiaPaCa-2 tumors treated with BEV (B) was reduced compared to the control (A). In contrast, there was no difference between Panc-1 tumors treated with BEV (D) and control (C). Scale bars: $200 \mu \mathrm{m}$. (E and F) Bar graphs of MVD in pancreatic tumors with and without BEV treatment. BEV significantly reduced the MVD of MiaPaCa- 2 compared to the control ( $p=$ 0.002) (E), but did not reduce the MVD of Panc-1 (F). ** p $<0.01$. 
was detected in the MiaPaCa-2 tumor after BEV $\rightarrow S$. typhimurium A1-R treatment (Fig. 5). Our data suggest that $S$. typhimurium A1-R is able to survive and multiply even in the hypo-vascular area of the tumor treated with $\mathrm{BEV}$ and cause tumor shrinkage.

To further demonstrate the advantages of $S$. typhimurium A1-R treatment following anti-VEGF therapy in the orthotopic mouse model, 20 mice with MiaPaCa-2-GFP orthotopic tumors were established and randomized to 4 groups: $\left(\mathrm{G}_{1}\right)$ saline (vehicle/control, 4 weeks); $\left(\mathrm{G}_{2}\right)$ GEM (4 weeks); $\left(\mathrm{G}_{3}\right)$ BEV (4 weeks) / GEM $(4$ weeks $)$ and $\left(\mathrm{G}_{4}\right)$ BEV (2 weeks) / GEM (2 weeks) $\rightarrow S$. typhimurium A1-R (2 weeks). Tumors were imaged and weighed after treatment (Fig. 6A). A large primary tumor and many metastases spreading over the entire abdominal cavity occurred in the control group (Fig. 6B; $\mathrm{G}_{1}$ ). Many metastases were found in the mice treated with GEM $\left(\mathrm{G}_{2}\right)$ (Fig. 6B), but were rarely found in the BEV/GEM $\left(\mathrm{G}_{3}\right)$ (Fig. 6B) and BEV/GEM $\rightarrow$ S. typhimurium A1-R groups $\left(\mathrm{G}_{4}\right)$ (Fig. 6B; $\left.\mathrm{G}_{4}\right)$. The mean tumor weight of each group in the MiaPaCa-2-GFP model was as follows: $\left(\mathrm{G}_{1}\right)$ Control: $2655.4 \pm 583.9 \mathrm{mg} ;\left(\mathrm{G}_{2}\right) \mathrm{GEM}$ : $775.9 \pm 273.8$ mg; $\left(\mathrm{G}_{3}\right)$ BEV/GEM: $413.5 \pm 108.3 \mathrm{mg} ;\left(\mathrm{G}_{4}\right) \mathrm{BEV} / \mathrm{GEM}$ $\rightarrow$ S. typhimurium A1-R: $257.5 \pm 57.1 \mathrm{mg}$. All regimens significantly reduced tumor weight compared to the control group $\left(\mathrm{G}_{2}: \mathrm{p}<0.001 ; \mathrm{G}_{3}: \mathrm{p}<0.001 ; \mathrm{G}_{4}: \mathrm{p}=0.001\right)$. $\mathrm{BEV} / \mathrm{GEM}$ significantly reduced tumor weight compared to GEM ( $\mathrm{p}=0.038)$. BEV/GEM $\rightarrow$ S. typhimurium A1-R significantly reduced tumor weight compared to GEM $(\mathrm{p}=0.012)$ and BEV/GEM $(\mathrm{p}=0.029)$. These results demonstrate that BEV/GEM $\rightarrow$ S. typhimurium A1-R sequential combination therapy is more effective than the $\mathrm{BEV} / \mathrm{GEM}$ combination.

\section{S. typhimurium A1-R following anti-VEGF therapy is effective in a VEGF-positive PDOX model}

Our laboratory pioneered surgical orthotopic implantation (SOI) metastatic mouse models of patient tumor specimens in the early 1990s [18-24]. These patient -derived orthotopic xenograft (PDOX) mouse models are more patient-like, especially with regard to metastasis, than ectopic subcutaneous models $[25,26]$.

A pancreatic cancer PDOX model was used to

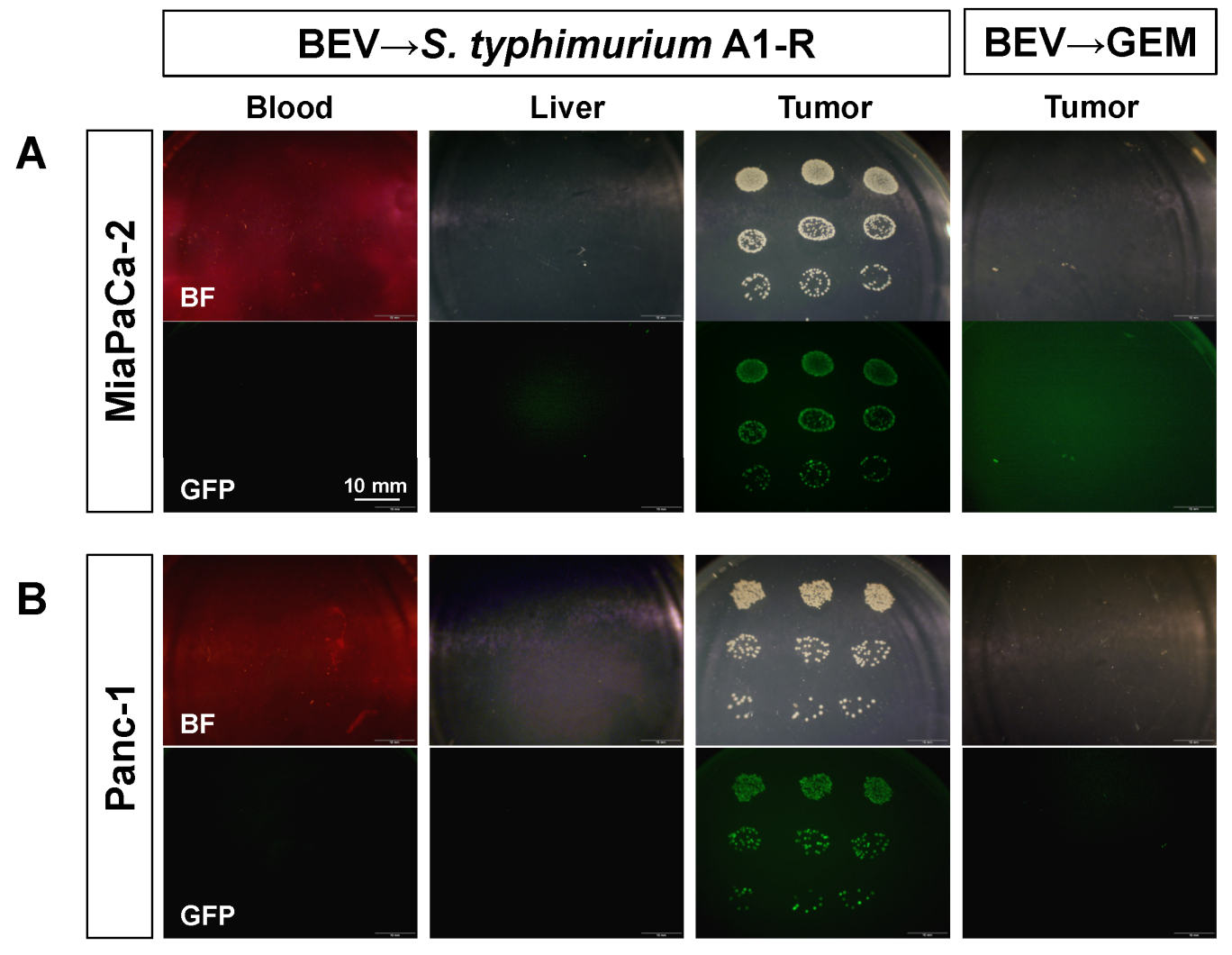

Figure 5: Distribution of GFP-labeled $S$. typhimurium A1-R bacteria in tumors and organs. Livers and tumors were minced and mixed with PBS, as was blood. The PBS, was plated on LB agar to identify S. typhimurium A1-R GFP in each tissue. Fluorescent $S$. typhimurium A1-R colonies were observed with the OV100 Small Animal Imaging System (Olympus Corp., Tokyo, Japan). Representative images of GFP-labeled S. typhimurium A1-R cultured from the tumor and the normal organs (blood and liver) of the mice in the BEV $\rightarrow S$. typhimurium A1-R and BEV $\rightarrow$ GEM groups. GFP-labeled S. typhimurium A1-R bacteria were clearly detected in both MiaPaCa-2 tumors (A) and Panc-1 tumors (B) in the BEV $\rightarrow$ S. typhimurium A1-R groups. No GFP-labeled S. typhimurium A1-R was detected in other tissues. Scale bars: $10 \mathrm{~mm} . \mathrm{BF}=$ brightfield. 
determine the efficacy of S. typhimurium A1-R treatment following anti-VEGF therapy. The patient tumor was a moderately-differentiated adenocarcinoma which expressed VEGF (Fig. 7A). Twenty mice with PDOXs were established and randomized to 4 groups and treated in the same way as the GFP MiaPaCa-2 orthotopic model. A large primary tumor and some metastases occurred in the control group. A few metastases were found in the

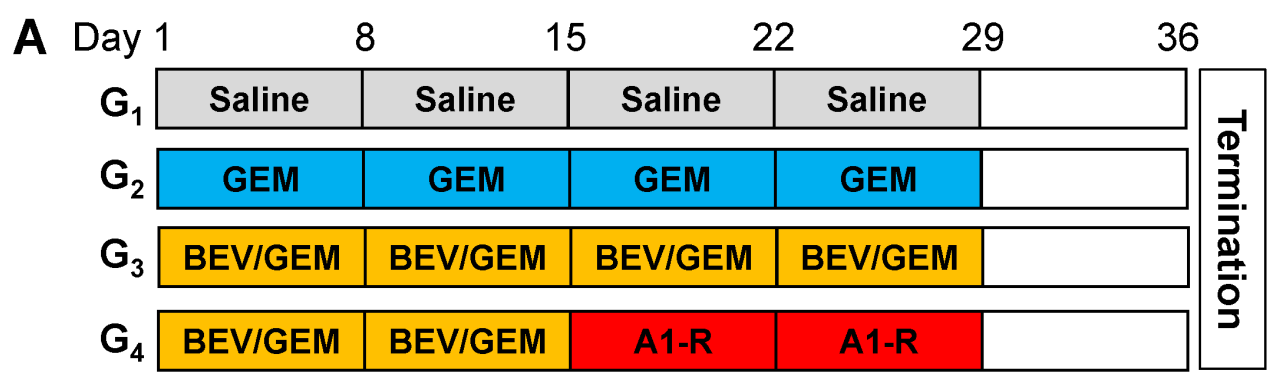

B
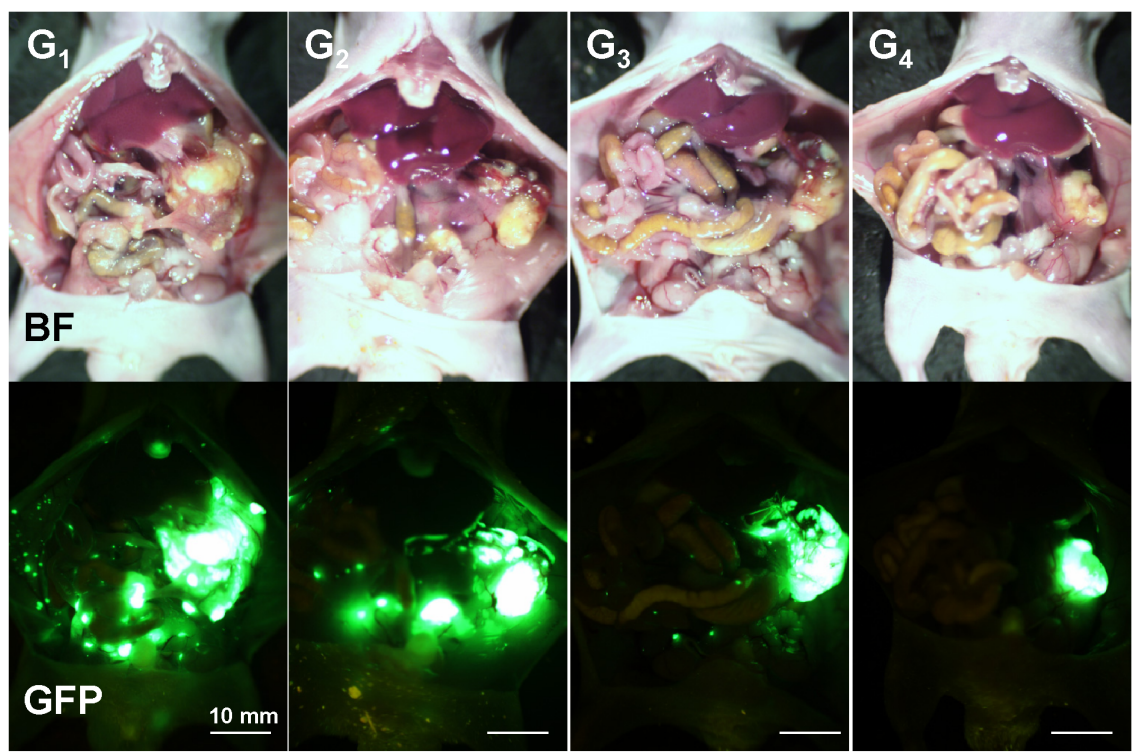

C

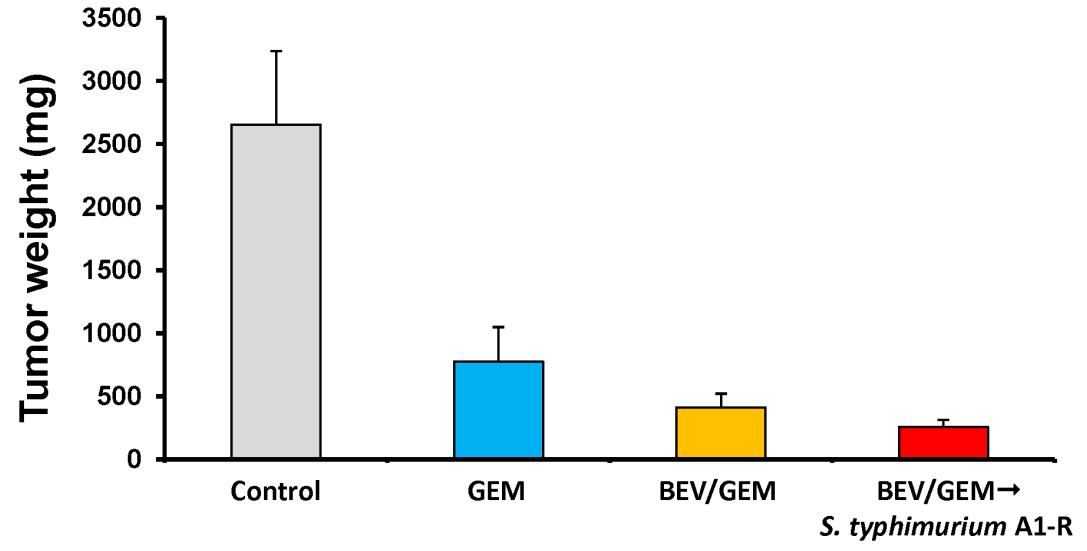

Figure 6: $S$. typhimurium A1-R treatment following BEV and GEM combination therapy of the MiaPaCa-2-GFP tumor in an orthotopic mouse model. (A) Schema of treatment of orthotopic MiaPaCa-2-GFP tumors. After confirmation of tumor growth by imaging with the OV100, the mice with orthotopic tumors were randomized to 4 groups: $\left(G_{1}\right)$ saline (vehicle/control, 4 weeks); $\left(G_{2}\right)$ GEM $(4$ weeks); $\left(\mathrm{G}_{3}\right)$ BEV (4 weeks) / GEM (4 weeks); and $\left(\mathrm{G}_{4}\right)$ BEV (2 weeks) / GEM (2 weeks) $\rightarrow$ S. typhimurium A1-R (2 weeks). Animals underwent laparotomy on day 36 , and the tumors were imaged with the OV100 and weighed and harvested for analysis. (B) Representative images at laparotomy. Upper panels indicate bright-field images (BF) and lower panels indicate GFP fluorescence images. A large primary tumor and many metastases spreading over the entire abdominal cavity were detected in the control group. Many metastases were found in the mice treated with GEM but rarely found in the BEV/GEM and BEV/GEM $\rightarrow$ S. typhimurium A1-R groups. All regimens significantly reduced tumor weight compared to the untreated control group $\left(\mathrm{G}_{2}: \mathrm{p}<0.001 ; \mathrm{G}_{3}: \mathrm{p}<0.001 ; \mathrm{G}_{4}: \mathrm{p}=0.001\right)$. BEV/GEM significantly reduced tumor weight compared to GEM $(\mathrm{p}=0.038)$. BEV/GEM $\rightarrow$ S. typhimurium A1-R significantly reduced tumor weight compared to GEM $(\mathrm{p}=0.012)$ and BEV/GEM $(\mathrm{p}=0.029)$. 
mice treated with GEM but rarely in the BEV/GEM and $\mathrm{BEV} / \mathrm{GEM} \rightarrow$ S. typhimurium A1-R groups (Fig. 7B). The mean tumor weight of each group in the PDOX model was as follows: $\left(\mathrm{G}_{1}\right)$ Control: $998.8 \pm 377.7 \mathrm{mg} ;\left(\mathrm{G}_{2}\right)$ GEM: $263.1 \pm 129.1 \mathrm{mg} ;\left(\mathrm{G}_{3}\right)$ BEV/GEM: $65.9 \pm 41.9 \mathrm{mg} ;\left(\mathrm{G}_{4}\right)$ $\mathrm{BEV} / \mathrm{GEM} \rightarrow$ S. typhimurium A1-R: $21.9 \pm 6.2 \mathrm{mg}$. All regimens significantly reduced tumor weight compared to the control group $\left(\mathrm{G}_{2}: \mathrm{p}=0.004 ; \mathrm{G} 3: \mathrm{p}=0.002 ; \mathrm{G} 4\right.$ : $\mathrm{p}=0.001)$. BEV/GEM significantly reduced tumor weight compared to GEM ( $\mathrm{p}=0.005)$. BEV/GEM $\rightarrow S$. typhimurium A1-R significantly reduced the tumor weight compared to GEM $(p=0.001)$ and BEV/GEM $(p=0.029)$. The results demonstrated in the PDOX model, BEV/GEM $\rightarrow$ S. typhimurium A1-R sequential combination therapy was also more effective than the BEV/GEM combination.

Previously developed concepts and strategies of highly selective tumor-targeting [27-34] can take advantage of the $S$. typhimuium A1-R tumor targeting described in the present report.

\section{MATERIALS AND METHODS}

\section{Cell culture and establishment of a green fluorescent protein-labeled cancer cell line}

Human pancreatic cancer cell lines Panc-1, MiaPaCa-2 and Hs766T were maintained in Dulbecco's modified Eagle's medium (Invitrogen, Carlsbad, CA, USA). Human pancreatic cancer cell lines BxPC-3 and Capan-1 were maintained in RPMI-1640 (Invitrogen, Carlsbad, CA, USA). All cell lines were incubated at $37^{\circ} \mathrm{C}$ in a humidified atmosphere containing $5 \% \mathrm{CO}_{2}$. Each medium was supplemented with $10 \%$ fetal bovine serum, streptomycin, and penicillin (complete medium). The cells were collected after trypsinization and stained with trypan blue (Sigma-Aldrich, St. Louis, MO). Only viable cells which excluded trypan blue were counted
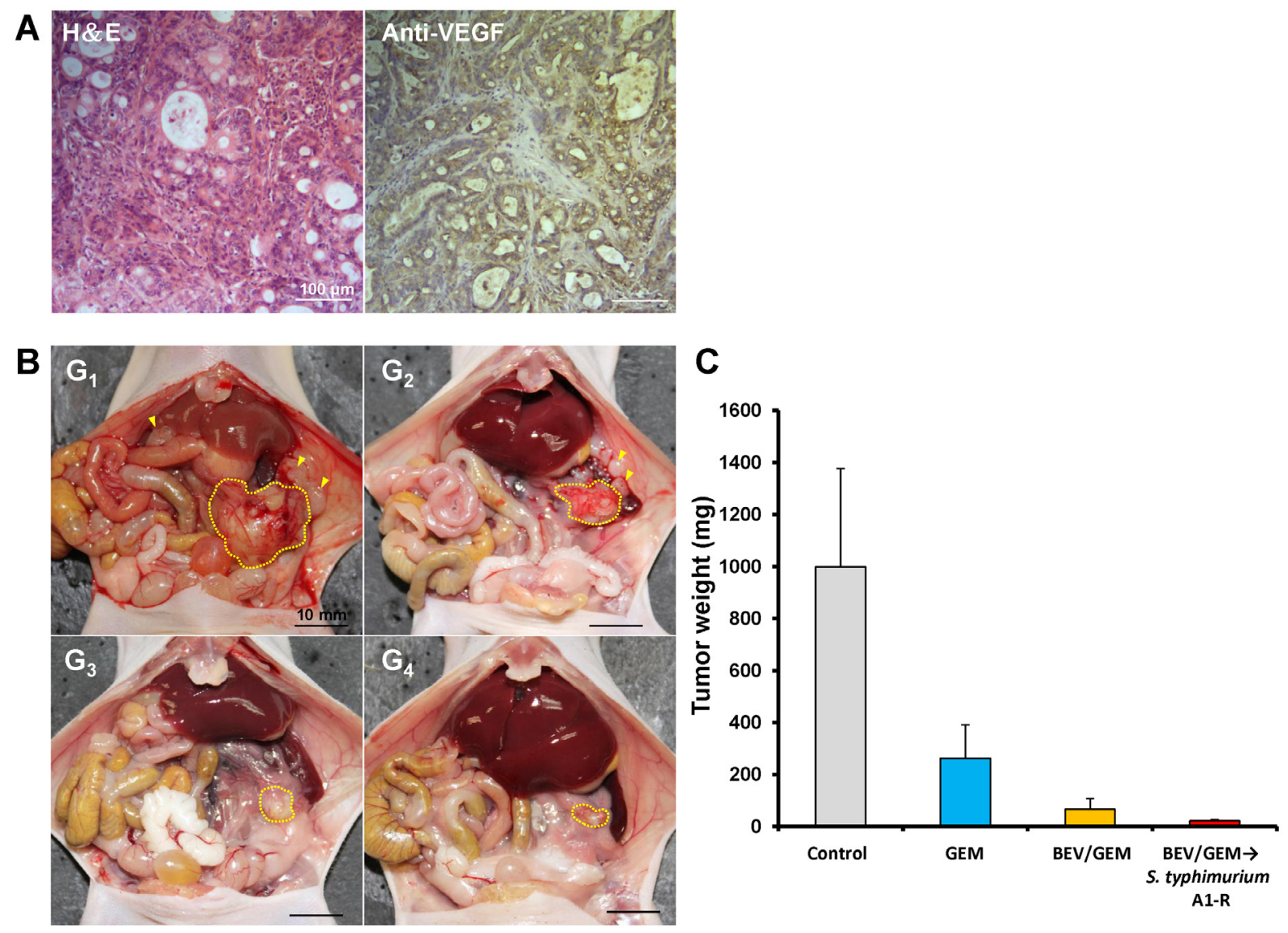

Figure 7: S. typhimurium A1-R treatment following BEV and GEM combination therapy on a VEGF-positive pancreatic cancer PDOX. (A) Histological characterization of the PDOX. The PDOX was diagnosed as moderately-differentiated adenocarcinoma with H\&E staining, and was strongly stained with an anti-VEGF antibody. Scale bars: $100 \mu \mathrm{m}$. (B) Representative images at laparotomy. The areas surrounded by the yellow broken lines indicate the primary tumors, and yellow arrow heads indicate metastasis. A large primary tumor and several metastases were detected in the control group $\left(\mathrm{G}_{1}\right)$. A few metastases were found in the mice treated with GEM $\left(\mathrm{G}_{2}\right)$ but rarely found in the $\mathrm{BEV} / \mathrm{GEM}\left(\mathrm{G}_{3}\right)$ and $\mathrm{BEV} / \mathrm{GEM} \rightarrow$ S. typhimurium $\mathrm{A} 1-\mathrm{R}$ groups $\left(\mathrm{G}_{4}\right)$. (C) Bar graphs of the total tumor weight (primary + metastasis) in each group. All regimens significantly reduced the tumor weight compared to the control group $\left(\mathrm{G}_{2}: \mathrm{p}=0.004 ; \mathrm{G}_{3}: \mathrm{p}=0.002 ; \mathrm{G}_{4}: \mathrm{p}=\right.$ $0.001)$. BEV/GEM significantly reduced tumor weight compared to GEM $(\mathrm{p}=0.005)$. BEV/GEM $\rightarrow$ S. typhimurium A1-R significantly reduced the tumor weight compared to GEM $(\mathrm{p}=0.001)$ and BEV/GEM $(\mathrm{p}=0.029)$. 
with a hemocytometer (Hausser Scientific, Horsham, PA). For GFP gene transduction of cancer cells, 70\% confluent human pancreatic cancer (MiaPaCa-2) cells were used. In brief, cells were incubated with a 1:1 precipitated mixture of retroviral supernatants of PT67-GFP packaging cells which express the GFP gene linked to the G418 resistance gene and RPMI 1640 (Irvine Scientific, Santa Ana, CA) containing $10 \%$ fetal bovine serum (FBS) (Hyclone Laboratories, Logan, UT) for 72 h. Fresh medium was replenished at this time. Cells were harvested with trypsin/ EDTA $72 \mathrm{~h}$ post-transduction and subcultured at a ratio of 1:15 into medium, which contained $200 \mu \mathrm{g} / \mathrm{ml}$ of the selective agent G418. The level of G418 was increased stepwise up to $800 \mu \mathrm{g} / \mathrm{ml}[35,36]$.

\section{Real-time RT-PCR}

Total RNA was extracted from human pancreatic cancer cell lines (BxPC-3, Capan-1, Hs766T, MiaPaCa-2 and Panc-1) using TRIzol (Invitrogen, Carlsbad, CA, USA), followed by on-column clean up with the RNA spin mini kit (GE Healthcare BioSciences, Little Chalfont, UK). Total RNA ( $2 \mu \mathrm{g})$ was reverse transcribed using the High Capacity RNA-to-cDNA kit (Applied Biosystems, Foster City, CA, USA) for complementary DNA (cDNA) synthesis. cDNA $(2 \mu \mathrm{l})$ in a final volume of $20 \mu \mathrm{l}$,was amplified using the following Taqman Gene Expression assays (Applied Biosystems): VEGFA (Hs00900055_m1); VEGFR1 (Hs01052961_m1); VEGFR2 (Hs00911700_ $\mathrm{m} 1)$; and glyceraldehyde-3-phosphate dehydrogenase $(G A P D H)$ endogenous control (Hs99999905_m1). All reactions were performed in triplicate using ABI 7900 HT Fast (Applied Biosystems). Analysis of relative gene expression data used the $\Delta \Delta \mathrm{Ct}$ method. An example is shown below:

$$
\begin{aligned}
& \text { Relative expression }=2^{-\Delta \Delta \mathrm{Ct}}, \Delta \Delta \mathrm{Ct}=\left(\mathrm{Ct},{ }_{\text {Target }}-\mathrm{Ct}\right. \text {, } \\
& \text { GAPDH })-\left(\mathrm{Ct},{ }_{\text {Panc- } 1}-\mathrm{Ct},{ }_{\mathrm{GAPDH}}\right) .
\end{aligned}
$$

\section{Preparation of bacteria}

S. typhimurium A1-R was grown overnight on LB medium and then diluted 1:10 in LB medium. Bacteria were harvested at late-log phase, washed with PBS, and then diluted in PBS for use in experiments [6].

\section{Confocal imaging of cancer cells infected with $S$. typhimurium A1-R in vitro}

Both MiaPaCa-2 and Panc-1 pancreatic cancer cell lines were infected with $S$. typhimurium A1-R GFP in vitro. Pancreatic cancer cells were grown in 24-well tissue culture plates to a density of approximately $10^{4}$ cells/well. S. typhimurium A1-R GFP were grown to late $\log$ in LB broth, diluted in cell culture medium and added to the cancer cells $\left(1 \times 10^{7} \mathrm{CFU} / \mathrm{ml}\right)$ and incubated at $37^{\circ} \mathrm{C}$. After $40 \mathrm{~min}$, the cells were rinsed and cultured in medium containing gentamycin sulfate $(20 \mu \mathrm{g} / \mathrm{ml})$ to kill external, but not internal bacteria. The interaction of $S$. typhimurium A1-R GFP with cancer cells in vitro was observed with confocal microscopy (Fluoview FV1000, Olympus, Tokyo, Japan). The excitation source was a semiconductor laser at $473 \mathrm{~nm}$ for GFP. Fluorescence images were obtained using the 20x/1.0 XLUMPLFLN objective [37].

\section{Clonogenic assay}

MiaPaCa-2 and Panc- 1 cells $\left(1 \times 10^{3}\right)$ were seeded in $35 \mathrm{~mm}$ dishes. S. typhimurium A1-R $\left(1 \times 10^{7} \mathrm{CFU} / \mathrm{ml}\right)$ was added to the cancer cells and incubated at $37^{\circ} \mathrm{C}$. After $40 \mathrm{~min}$, the cells were rinsed and cultured in medium containing gentamycin sulfate $(20 \mu \mathrm{g} / \mathrm{ml})$. After 7 -days culture, the cancer-cell colonies were fixed with ethanol and then stained with crystal violet. ImageJ was used to quantify the cell colonies.

\section{Animals}

Male athymic (nu/nu) nude mice (AntiCancer, Inc., San Diego) (4-6 weeks) were used in this study. Mice were kept in a barrier facility under HEPA filtration. Mice were fed with autoclaved laboratory rodent diet. All surgical procedures and imaging were performed with the animals anesthetized by intramuscular injection of a solution of $50 \%$ ketamine, 38\% xylazine, and $12 \%$ acepromazine maleate $(0.02 \mathrm{ml})$. All animal studies were conducted in accordance with the principals and procedures outlined in the NIH Guide for the Care and Use of Laboratory Animals under PHS Assurance Number A3873-1.

\section{Subcutaneous pancreatic cancer cell implantation}

Panc-1 and MiaPaCa-2 pancreatic cancer cells were harvested by trypsinization and washed twice with serum-free medium. Cells $\left(2 \times 10^{6}\right.$ in $100 \mu \mathrm{l}$ serum-free medium) were injected subcutaneously within $30 \mathrm{~min}$ of harvesting, over the right and left flanks in male nude mice. Subcutaneous tumors were allowed to grow for 2-4 weeks until large enough for subsequent experiments or orthotopic implantation.

\section{Assessment of microvessel density (MVD) in xenograft tumors}

Frozen tumor sections $(7 \mu \mathrm{m})$ were fixed with methanol. The sections were then treated for $30 \mathrm{~min}$ with hydrogen peroxide $(0.3 \%)$ to block endogenous peroxidase activity. After incubation with normal goat 
serum $15 \%$, the sections were incubated with anti-mouse CD31 (1:100; BD Pharmigen, San Jose, CA, USA) for 1 hour at room temperature. The primary antibodies were detected using anti-rat secondary antibodies and avidin/biotin/horseradish peroxidase complex (Vector Laboratories, Burlingame, CA, USA) for $30 \mathrm{~min}$ at room temperature. The labeled antigens were visualized with the DAB kit (DAKO Cytomation, Kyoto, Japan). The sections were counterstained with hematoxylin and examined using a $\mathrm{BH}-2$ microscope (Olympus) equipped with an INFINITY1 2.0 megapixel CMOS digital camera (Lumenera Corporation, Ottawa, Canada). All images were acquired using INFINITY ANALYZE software (Lumenera Corporation) without post-acquisition processing. MVD was determined by counting three fields at $\times 100$ magnification of the highest vascular density.

\section{Specimen collection}

Patient pancreatic tumor samples were procured with informed written consent and the study was conducted under the approval of the Institutional Review Board of the UC San Diego Medical Center.

\section{Orthotopic tumor implantation}

A small transverse incision (6- to 10-mm) was made on the left flank of the mouse through the skin and peritoneum. The tail of the pancreas was exposed through this incision, and a single tumor fragment (3$\mathrm{mm}^{3}$ ) harvested from a subcutaneous tumor was sutured to the tail of the pancreas using 8-0 nylon surgical sutures (Ethilon; Ethicon Inc., NJ, USA). On completion, the tail of the pancreas was returned to the abdomen, and the incision was closed in one layer using 6-0 nylon surgical sutures [19, 24].

\section{Treatment}

Mice were randomized to 4 groups and treated as follows: $\left(\mathrm{G}_{1}\right)$ saline (vehicle/control, ip, weekly, 4 weeks); $\left(\mathrm{G}_{2}\right)$ GEM (Eli Lilly, Indianapolis, IN) (80 mg/ $\mathrm{kg}$, ip, weekly, 4 weeks); $\left(\mathrm{G}_{3}\right)$ BEV (Genentech, South San Francisco, CA)(5 mg/kg, ip, twice a week, 4 weeks $)$ / GEM (80 mg/kg, ip, weekly, 4 weeks) and $\left(\mathrm{G}_{4}\right)$ BEV (5 mg/kg, ip, twice a week, 2 weeks) / GEM (80 mg/ $\mathrm{kg}$, ip, weekly, 2 weeks) $\rightarrow$ S. typhimurium A1-R (5 $\times$ $10^{7} \mathrm{CFU} /$ body, iv, weekly, 2 weeks) (Fig. 4A). Each treatment group comprised 5 tumor-bearing mice. Body weights of the mice were determined on a balance once a week. Tumor size was measured with calipers in the subcutaneous models, and at laparotomy in the orthotopic models on day 36. Tumors were imaged with an OV100 Small Animal Imaging System (Olympus, Tokyo, Japan) or a Canon EOS 60D digital camera with an EF-S18-55 IS lens (Canon, Tokyo, Japan) and weighed and harvested for analysis.

\section{Tissue histology}

Tumor samples were removed with surrounding normal tissues at the time of resection. Fresh tissue samples were fixed in $10 \%$ formalin and embedded in paraffin before sectioning and staining. Tissue sections (3 $\mu \mathrm{m})$ were deparaffinized in xylene and rehydrated in an ethanol series. Hematoxylin and eosin (H \& E) staining was performed according to standard protocols.

\section{Fluorescence in vivo imaging}

The OV100 Small Animal Imaging System, containing an MT-20 light source (Olympus Biosystems, Planegg, Germany) and DP70 CCD camera (Olympus) were used for imaging GFP-labeled S. typhimurium A1-R and orthotopic tumors in live mice [38].

\section{Detection of GFP-labeled S. typhimurium A1-R bacteria in tumors and organs}

Tissues from subcutaneous tumors and normal organs (blood, spleen and liver) were removed at termination from the nude mice with subcutaneous tumors. S. typhimurium A1-R was extracted from the tumors and organs and cultured in LB agar for 24 hours, and imaged with the Olympus OV100.

\section{Statistical analysis}

PASW Statistics 18.0 (SPSS, Inc) was used for all statistical analyses. The Student's $t$-test was used to compare continuous variables between two groups. Analysis of variance models were used to compare multiple groups. A $p$ value of $\leq 0.05$ was considered statistically significant for all comparisons.

\section{ACKNOWLEDGEMENTS}

This study was supported in part by National Cancer Institute grants CA132971 and 142669 (to M.B. and AntiCancer, Inc.) and CA183280 (to AntiCancer, Inc.) and JSPS KAKENHI Grant Numbers 26830081 to Y.H., 26462070 to I.E and 24592009 to K.T.

\section{DEDICATION}

This paper is dedicated to the memory of A. R. Moossa, M.D. 


\section{CONFLICTS OF INTEREST}

Y.Z. and M.Z. are employees of AntiCancer Inc. Y.H., T.M., S.M., M.Y., S.Y. and R.M.H. are unsalaried associates of AntiCancer Inc. There are no other competing financial interests.

\section{REFERENCES}

1. Hidalgo M. Pancreatic cancer. New Engl J Med 2010; 362:1605-17.

2. Seo Y, Baba H, Fukuda T, Takashima M, Sugimachi K. High expression of vascular endothelial growth factor is associated with liver metastasis and a poor prognosis for patients with ductal pancreatic adenocarcinoma. Cancer 2000; 88:2239-45.

3. Niedergethmann M, Hildenbrand R, Wostbrock B, Hartel M, Sturm JW, Richter A, Post S. High expression of vascular endothelial growth factor predicts early recurrence and poor prognosis after curative resection for ductal adenocarcinoma of the pancreas. Pancreas 2002; 25:122-9.

4. Tamburrino A, Piro G, Carbone C, Tortora G, Melisi D. Mechanisms of resistance to chemotherapeutic and antiangiogenic drugs as novel targets for pancreatic cancer therapy. Front Pharmacol 2013; 4:56.

5. Zhao M, Yang M, Li XM, Jiang P, Baranov E, Li S, Xu M, Penman S, Hoffman RM. Tumor-targeting bacterial therapy with amino acid auxotrophs of GFP-expressing Salmonella typhimurium. Proc Natl Acad Sci USA 2005; 102:755-60.

6. Zhao M, Yang M, Ma H, Li X, Tan X, Li S, Yang Z, Hoffman RM. Targeted therapy with a Salmonella typhimurium leucine-arginine auxotroph cures orthotopic human breast tumors in nude mice. Cancer Res 2006; 66:7647-52.

7. Zhao M, Geller J, Ma H, Yang M, Penman S, Hoffman RM. Monotherapy with a tumor-targeting mutant of Salmonella typhimurium cures orthotopic metastatic mouse models of human prostate cancer. Proc Natl Acad Sci USA 2007; 104:10170-4.

8. Zhang Y, Tome Y, Suetsugu A, Zhang L, Zhang N, Hoffman RM, Zhao M. Determination of the optimal route of administration of Salmonella typhimurium A1-R to target breast cancer in nude mice. Anticancer Res 2012; 32:2501-8.

9. Nagakura C, Hayashi K, Zhao M, Yamauchi K, Yamamoto N, Tsuchiya H, Tomita K, Bouvet M, Hoffman RM. Efficacy of a genetically-modified Salmonella typhimurium in an orthotopic human pancreatic cancer in nude mice. Anticancer Res 2009; 29:1873-8.

10. Yam C, Zhao M, Hayashi K, Ma H, Kishimoto H, McElroy M, Bouvet M, Hoffman RM. Monotherapy with a tumortargeting mutant of S. typhimurium inhibits liver metastasis in a mouse model of pancreatic cancer. J Surg Res 2010; 164:248-55.
11. Hiroshima Y, Zhao M, Zhang Y, Maawy A, Hassanein MK, Uehara F, Miwa S, Yano S, Momiyama M, Suetsugu A, et al. Comparison of efficacy of Salmonella typhimurium A1-R and chemotherapy on stem-like and non-stem human pancreatic cancer cells. Cell Cycle 2013; 12:2774-80.

12. Hiroshima Y, Zhao M, Maawy A, Zhang Y, Katz MH, Fleming JB, Uehara F, Miwa S, Yano S, Momiyama M, et al. Efficacy of Salmonella typhimurium A1-R versus chemotherapy on a pancreatic cancer patient-derived orthotopic xenograft (PDOX). J Cell Biochem 2014; 115:1254-61.

13. Kimura H, Zhang L, Zhao M, Hayashi K, Tsuchiya H, Tomita K, Bouvet M, Wessels J, Hoffman RM. Targeted therapy of spinal cord glioma with a genetically-modified Salmonella typhimurium. Cell Prolif 2010; 43:41-8.

14. Momiyama M, Zhao M, Kimura H, Tran B, Chishima T, Bouvet M, Endo I, Hoffman RM. Inhibition and eradication of human glioma with tumor-targeting Salmonella typhimurium in an orthotopic nude-mouse model. Cell Cycle 2012; 11:628-32.

15. Zhao M, Suetsugu A, Ma H, Zhang L, Liu F, Zhang Y, Tran B, Hoffman RM. Efficacy against lung metastasis with a tumor-targeting mutant of Salmonella typhimurium in immunocompetent mice. Cell Cycle 2012; 11187-93.

16. Hayashi K, Zhao M, Yamauchi K, Yamamoto N, Tsuchiya H, Tomita K, Hoffman RM. Cancer metastasis directly eradicated by targeted therapy with a modified Salmonella typhimurium. J Cell Biochem 2009; 106:992-8.

17. Hayashi K, Zhao M, Yamauchi K, Yamamoto N, Tsuchiya H, Tomita K, Kishimoto H, Bouvet M, Hoffman RM. Systemic targeting of primary bone tumor and lung metastasis of high-grade osteosarcoma in nude mice with a tumor-selective strain of Salmonella typhimurium. Cell Cycle 2009; 8:870-5.

18. Fu X, Besterman JM, Monosov A, Hoffman RM. Models of human metastatic colon cancer in nude mice orthotopically constructed by using histologically intact patient specimens. Proc Natl Acad Sci USA 1991; 88:9345-9.

19. Fu X, Guadagni F, Hoffman RM. A metastatic nudemouse model of human pancreatic cancer constructed orthotopically from histologically intact patient specimens. Proc Natl Acad Sci USA 1992; 89:5645-9.

20. Wang X, Fu X, Hoffman RM. A new patient-like metastatic model of human lung cancer constructed orthotopically with intact tissue via thoracotomy in immunodeficient mice. Int J Cancer 1992; 51:992-5.

21. Fu X, Hoffman RM. Human ovarian carcinoma metastatic models constructed in nude mice by orthotopic transplantation of histologically-intact patient specimens. Anticancer Res 1993; 13:283-6.

22. Fu X, Le P, Hoffman RM. A metastatic-orthotopic transplant nude mouse model of human patient breast cancer. Anticancer Res 1993; 13:901 4.

23. Furukawa T, Kubota T, Watanabe M, Kitajima M, Fu X, 
Hoffman RM. Orthotopic transplantation of histologically intact clinical specimens of stomach cancer to nude mice: correlation of metastatic sites in mouse and individual patient donors. Int J Cancer 1993; 53:608 12.

24. Hoffman RM. Orthotopic metastatic mouse models for anticancer drug discovery and evaluation: a bridge to the clinic. Investigational New Drugs 1999; 17:343-59.

25. Bertotti A, Migliardi G, Galimi F, Sassi F, Torti D, Isella C, Cora D, Di Nicolantonio F, Buscarino M, Petti C, et al. A molecularly annotated platform of patient-derived xenografts ("xenopatients") identifies HER2 as an effective therapeutic target in cetuximab-resistant colorectal cancer. Cancer Discov 2011; 1:508-23.

26. Talmadge JE, Singh RK, Fidler IJ, Raz A. 2007. Murine models to evaluate novel and conventional therapeutic strategies for cancer. Am J Path 2007; 170:793-804.

27. Blagosklonny MV. How cancer could be cured by 2015. Cell Cycle 2005; 4:269-78.

28. Blagosklonny MV. Tissue-selective therapy of cancer. Br J Cancer 2003; 89:1147-51.

29. Blagosklonny MV. Matching targets for selective cancer therapy. Drug Discov Today 2003; 8:1104-7.

30. Blagosklonny MV. "Targeting the absence" and therapeutic engineering for cancer therapy. Cell Cycle 2008; 7:1307-12.

31. Blagosklonny MV. Teratogens as anti-cancer drugs. Cell Cycle 2005; 4:1518-21.

32. Blagosklonny MV. Treatment with inhibitors of caspases, that are substrates of drug transporters, selectively permits chemotherapy-induced apoptosis in multidrug-resistant cells but protects normal cells. Leukemia 2001; 15:936-41.

33. Blagosklonny MV. Target for cancer therapy: proliferating cells or stem cells. Leukemia 2006; 20:385-91.

34. Blagosklonny MV. Cancer stem cell and cancer stemloids: from biology to therapy. Cancer Biol Ther 2007; 6:1684-90.

35. Bouvet M, Tsuji K, Yang M, Jiang P, Moossa AR, Hoffman RM. 2006. In vivo color-coded imaging of the interaction of colon cancer cells and splenocytes in the formation of liver metastases. Cancer Res 2006; 66:11293-7.

36. Hoffman RM, Yang M. Subcellular imaging in the live mouse. Nat Protoc 2006; 1:775-82.

37. Uchugonova A, Duong J, Zhang N, König K, Hoffman $\mathrm{RM}$. The bulge area is the origin of nestin-expressing pluripotent stem cells of the hair follicle. J Cell Biochem 2011; 112:2046-50.

38. Yamauchi K, Yang M, Jiang $\mathrm{P}, \mathrm{Xu} \mathrm{M}$, Yamamoto N, Tsuchiya H, Tomita K, Moossa AR, Bouvet M, Hoffman RM. Development of real-time subcellular dynamic multicolor imaging of cancer cell trafficking in live mice with a variable-magnification whole-mouse imaging system. Cancer Res 2006; 66:4208-14. 